\title{
Experimental Investigation of Beam Breakup in the Jefferson Laboratory 10 kW FEL Upgrade Driver*
}

\author{
C. Tennant ${ }^{\#}$, D. Douglas, K. Jordan, L. Merminga, E. Pozdeyev, H. Wang \\ Thomas Jefferson National Accelerator Facility, Newport News, VA, 23606 \\ T.I. Smith, W.W. Hansen Experimental Physics Laboratory, Stanford, CA 94305 \\ I.V. Bazarov, G. Hoffstaetter, Cornell University, Ithaca, NY 14850 \\ Stefan Simrock, DESY, Hamburg, Germany
}

\section{Abstract}

In recirculating accelerators, and in particular energy recovery linacs (ERLs), the maximum current has been limited by multipass, multibunch beam breakup (BBU), which occurs when the electron beam interacts with the higher-order modes (HOMs) of an accelerating cavity on the accelerating pass and again on the energy recovered pass. This effect is of particular concern in the design of modern high average current energy recovery accelerators utilizing superconducting RF technology. Experimental observations of the instability at the Jefferson Laboratory $10 \mathrm{~kW}$ Free-Electron Laser (FEL) are presented. Measurements of the threshold current for the instability are presented and compared to the predictions of several BBU simulation codes. With BBU posing a threat to high current beam operation in the FEL Driver, several suppression schemes were developed. These include direct damping of the dangerous HOMs and appropriately modifying the electron beam optics. Preliminary results of their effectiveness in raising the threshold current for stability are presented.

\section{INTRODUCTION}

For a two-pass accelerator with a single accelerating cavity containing a single dipole HOM with arbitrary polarization, $\alpha$, the transverse BBU threshold current is given by the expression

$$
I_{t h}=-\frac{2 c p_{b}}{e M^{*}(R / Q) Q k \sin \left(\omega T_{r}\right)}
$$

where

$M^{*} \equiv M_{12} \cos ^{2} \alpha+\left(M_{14}+M_{32}\right) \sin \alpha \cos \alpha+M_{34} \sin ^{2} \alpha$

and $p_{b}$ is the beam momentum at the cavity, $(R / Q) Q$ is the impedance of the mode, $k$ is the wavenumber and $T_{r}$ is the recirculation time [1]. This model disregards the degeneracy of the dipole HOMs. For the Jefferson Laboratory FEL Driver this is a valid assumption. Due to mechanical errors and other perturbations introduced during the fabrication of the cavities, the modes are separated by several bandwidths in frequency.

\section{BBU CHARACTERIZATION}

The primary goal of the BBU studies was to characterize the instability to the extent that $\mathrm{BBU}$ simulation codes could be satisfactorily benchmarked.

\footnotetext{
Supported by the Office of Naval Research, the Joint Technology Office, the Commonwealth of Virginia, the Air Force Research Laboratory, and by DOE Contract DE-AC05-84ER40150.

\# tennant@jlab.org
}

\section{Experimental Setup}

The key element in the measurements used to characterize BBU was the fact that the unstable cryomodule, unlike previous CEBAF modules, uses DESY-like coaxial HOM couplers. This allows us to individually monitor the HOM power (via Schottky diodes) from each of the 8 cavities. Using four 4-channel oscilloscopes to monitor the HOM power from each HOM coupler port in all 8 cavities, one can easily identify the cavity containing the unstable mode with a cursory glance of the oscilloscope traces by noting any exponential growth in power. Upon identifying the cavity containing the unstable mode, the next step is to identify the frequency of the mode. This is accomplished by bypassing the Schottky diode and measuring the HOM voltage directly during a beam breakup. Taking the FFT of the voltage signal yields the HOM frequency causing BBU. The results of our measurements indicate that with nominal optics for an $88 \mathrm{MeV}$ machine configuration, the most dangerous mode is the $2106 \mathrm{MHz}$ HOM located in cavity 7 . This agrees with simulation results which predict this mode is highly unstable. Having identified the mode, measurements of the threshold current can begin.

\section{Measuring the Threshold Current}

Given a description of the machine optics and HOM parameters (frequency, $Q_{L}, R / Q$ ) BBU simulation codes calculate a threshold current. Hence the easiest way to benchmark the codes is to compare this threshold current with experimental measurements. Three different methods were used to measure the threshold current for a particular machine configuration. The first, if the threshold current was sufficiently low, was by direct observation. That is, noting the average beam current at which a BBU-induced machine trip occurs.

The remaining two methods make use of an important aspect of BBU, namely how the HOM voltage behaves above and below the threshold current. From the equation that describes the evolution of the voltage, one can extract an exceedingly useful quantity which we define as the effective quality factor

$$
Q_{e f f}=Q_{L} \frac{I_{t h}}{I_{t h}-I}
$$

This simple relation says that one needs only to measure the effective $Q$ as a function of the average beam current and in principle the threshold is easily extracted. Note that Eq. (2) is valid both above and below the threshold current. 
The beam-transfer function (BTF) measurement is the second method used to measure the threshold current and amounts to making an $\mathrm{S}_{21}$ measurement of a particular mode as a function of average beam current. By measuring the effective $Q$, that is the $Q$ of the HOM-beam system, as a function of current, one can use Eq. (2) to extract the threshold current.

The third and final measure of the threshold is achieved by measuring the growth rate of the HOM power. With this method the growth rate is described by the time constant for the HOM-beam system

$$
\tau_{\text {eff }}=\tau_{o} \frac{I_{t h}}{I_{t h}-I}
$$

which is an equivalent way of expressing Eq. (2). Similar to the BTF measurement, by measuring the effective $\tau$ as a function of current, one can use Eq. (3) to extract the threshold current. The method of measuring the HOM power and the BTF measurement combine to create a complementary set of measurements. Whereas measuring the power is a time-domain measurement made above the threshold current (and typically in pulsed-beam mode), the BTF measurement is inherently a frequency-domain measurement made below the threshold current (and typically with cw beam).

\section{BEAM OPTICAL SUPPRESSION}

With a comprehensive characterization of BBU complete, our attention is now turned towards exercising suppression techniques. Methods to manipulate the transverse beam optics in such a way as to suppress BBU were first discussed by Rand and Smith in 1980 [2]. Specifically, we demonstrated the ability to raise the threshold current by use of a phase trombone, a local reflector and a pseudo-reflector. For a more complete introduction and analysis of these beam optical techniques the reader is referred to references [2], [3], [4] and [5]; here only the results of each of these are discussed.

\section{Phase Trombone}

With a judicious change in the phase advance, one can achieve point-to-point focusing $\left(M_{12}\right.$ or $\left.M_{34}=0\right)$ at the location of an unstable mode so that an HOM-induced kick on the first pass results in a zero displacement on the second pass [6]. By all indications the dangerous 2106 $\mathrm{MHz} \mathrm{HOM}$ is a vertically polarized mode. To change the vertical phase advance, the strength of four quadrupoles in the recirculator were changed from their nominal setpoint in steps of $100 \mathrm{G}$ from $-200 \mathrm{G}$ to $+300 \mathrm{G}$ while measuring the threshold current at each point. The effect was to decrease the threshold current from below $1 \mathrm{~mA}(-200 \mathrm{G})$ to stabilizing the mode $(+300 \mathrm{G})$. The effect of changing the quadrupoles from $+200 \mathrm{G}$ to $+300 \mathrm{G}$ is illustrated by the BTF measurement data in Fig. 1. The fact that the mode was eventually stabilized ( $Q_{\text {eff }}$ decreases as the current increases) indicates that the sign of the $M_{34}$ element of the recirculation matrix from the unstable cavity back to itself had been changed.
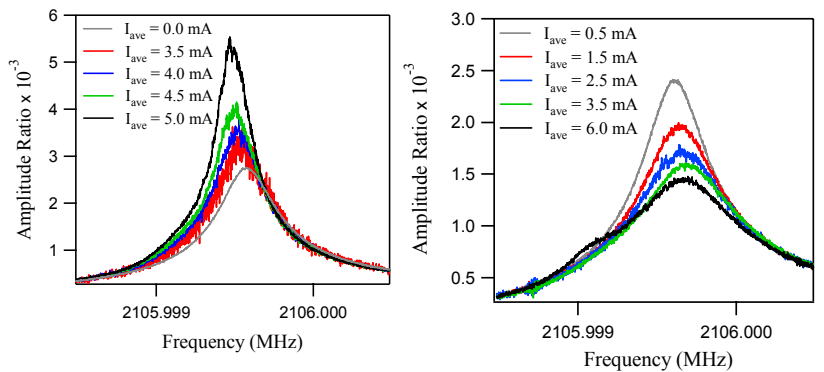

Figure 1: BTF results with the phase trombone for $+200 \mathrm{G}$ (left) and $+300 \mathrm{G}$ (right).

\section{Local Reflector}

The idea behind implementing a local reflector is to map a BBU-induced horizontal kick into the vertical plane, and likewise to map a BBU-induced vertical kick to the horizontal plane. A practical implementation of a local reflector using skew-quadrupoles has been non-invasively embedded in the $3 \mathrm{~F}$ region of the FEL Upgrade Driver [7]. To experimentally measure the effect of the reflector, we first established the threshold current without the reflector to be $1.8 \mathrm{~mA}$ by direct observation. The reflector was then activated and a BTF measurement was performed for the $2106 \mathrm{MHz}$ mode. The results of the measurement yielded a new threshold current of $9 \mathrm{~mA}-$ an increase by a factor of 5 .

\section{Pseudo-Reflector}

The term pseudo-reflector is used to describe a $4 \times 4$ recirculation transfer matrix from the unstable cavity back to itself that is completely coupled and where the offdiagonal $2 \times 2$ matrices are equal and of opposite sign. In some instances more effective damping can be achieved with such transverse optics. The existing local reflector embedded in the FEL Driver recirculator can, in principle, be made to produce such a transfer matrix [8].

Having generated what we believed to be a recirculation matrix that describes a pseudo-reflection, we followed the usual procedure of comparing the extrapolated threshold current from the BTF measurement with the threshold current with nominal beam optics. The result of the measurement for the $2106 \mathrm{MHz}$ mode with the pseudo-reflector activated indicates that the mode has been stabilized. Applying Eq. (2) to the data yields a threshold current of $-17 \mathrm{~mA}$ where a negative threshold is an indication of the strength of the stability.

\section{$Q$-DAMPING}

Damping the HOM quality factors is achieved primarily by HOM couplers. However, in the event that the HOMs need further damping, two methods have been developed and successfully exercised in the FEL Driver.

\section{Active Damping Circuit}

The idea of the damping circuit is as follows; couple power from one of the HOM ports and using a narrowband filter select the HOM frequency of interest, shift the signal by 180 degrees in phase, amplify the 
signal and then return it to the cavity through the same HOM port. Since the threshold current is inversely proportional to the $Q$ of the mode we expect that a factor of 5 decrease in the $Q$ will result in a corresponding increase in the threshold current. This is, in fact, what we observe experimentally. The threshold current was determined to be $2.3 \mathrm{~mA}$ with the damping circuit off by direct observation. With the damping circuit on a BTF measurement was performed for the $2106 \mathrm{MHz}$ mode with the results shown in Fig. 2. The two traces are due to a discrepancy between the two diagnostics used to measure the average beam current during the measurement. Therefore, depending on which diagnostic you trust, the threshold current is either $7.5 \mathrm{~mA}$ or 10.5 $\mathrm{mA}$. Nevertheless, we see that the threshold current scales approximately as expected with the $Q$ of the $2106 \mathrm{MHz}$ mode.

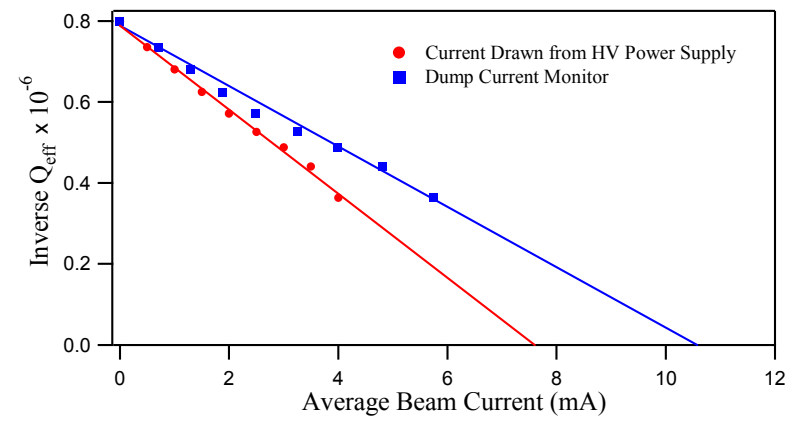

Figure 2: Analysis of BTF measurement with $Q$-damping circuit activated.

\section{3-Stub Tuner}

The 3-stub tuner acts as an impedance transformer. The idea is to manipulate the stubs in such a fashion that the incident HOM voltage is reflected with a 180 degree phase shift. Because the stub tuner is a passive device, the attenuation in the cables from the HOM ports prevented optimal $Q$-damping. Additionally, a broken connection in the cable leading to one of the HOM ports created unwanted reflections to the cavity and further decreased the effectiveness of the damping. Nevertheless, a modest decrease (a factor of 1.5) in the $Q$ of the $2106 \mathrm{MHz}$ mode and the corresponding increase in the threshold current were observed.

\section{SUMMARY OF SUPPRESSION TECHNIQUES}

Several methods for suppressing beam breakup have been successfully demonstrated in the FEL Driver. A summary of the techniques used and their effect on the $2106 \mathrm{MHz}$ mode are given in Table 1. First, note that the active damping methods work for only a single mode per cavity due to the fact that these methods require extracting a signal from the HOM coupler, of which there is one per cavity. It is clear that direct $Q$-damping can increase the threshold by orders of a few, but could not completely stabilize the mode. Nonetheless, the attractive feature of $Q$-damping is that it does not affect the beam optics. On the other hand, the beam optical suppression techniques were able to stabilize the $2106 \mathrm{MHz}$ mode against beam breakup. During the experimental investigations of BBU we were not concerned with making the FEL lase, thus one of the questions that remain is; does invoking beam optical suppression techniques prohibit one from achieving a machine configuration that is suitable for lasing?

\begin{tabular}{|l|c|}
\hline Suppression Technique & Effect \\
\hline Damping Circuit & $(3.3-4.6) \times \mathrm{I}_{\text {th }}$ \\
\hline 3-Stub Tuner & $1.6 \times \mathrm{I}_{\text {th }}$ \\
\hline Phase Trombone & Stabilized \\
\hline Local Reflector & $5 \times \mathrm{I}_{\text {th }}$ \\
\hline Psuedo-Reflector & Stabilized \\
\hline
\end{tabular}

Table 1: Summary of BBU suppression techniques and the effects on the threshold current.

\section{CONCLUSION}

The FEL Driver has proven to be a valuable tool in investigating both the beam breakup instability and methods of suppressing it. The primary goal of these studies was to characterize BBU to the extent that BBU simulation codes could be satisfactorily benchmarked. Initial results show excellent agreement between experimental data and the predictions from simulations. However, work is still being done to provide a more accurate description of the machine optics by analyzing difference orbit data. In addition to this work, two classes of suppression techniques were explored; (1) direct $Q$ damping and (2) beam optical schemes. It was demonstrated that both methods could be used to effectively increase the threshold current in the FEL Driver.

\section{ACKNOWLEDGEMENTS}

The authors gratefully acknowledge the entire Jefferson Laboratory FEL team for their support during these measurements. In addition we would like to thank Curt Hovater and Byung Yunn for their advice and assistance.

\section{REFERENCES}

[1] E. Pozdeyev, submitted to PRST-AB.

[2] R. Rand and T. Smith, Particle Accelerators, 11, 1, 1980.

[3] C. Tennant et. al., submitted to PRST-AB.

[4] To be published in Proceedings of $32^{\text {nd }}$ ICFA Workshop on ERLs, 2005.

[5] B. Yunn, JLAB Technical Report 05-032, 2005.

[6] D. Douglas, JLAB Technical Report 04-017, 2004.

[7] D. Douglas, JLAB Technical Report 04-016, 2004.

[8] D. Douglas, JLAB Technical Report 04-023, 2004. 